Meta

Journal des traducteurs

Translators' Journal

\title{
Computational Discourse Analysis for Interpretation
}

\section{Sane M. Yagi}

Volume 44, numéro 2, juin 1999

URI : https://id.erudit.org/iderudit/004627ar

DOI : https://doi.org/10.7202/004627ar

Aller au sommaire du numéro

Éditeur(s)

Les Presses de l'Université de Montréal

ISSN

0026-0452 (imprimé)

1492-1421 (numérique)

Découvrir la revue

Citer cet article

Yagi, S. M. (1999). Computational Discourse Analysis for Interpretation. Meta, 44(2), 268-279. https://doi.org/10.7202/004627ar

\section{Résumé de l'article}

Une m"thode informatis"e qui permet lõexamen attentif du discours dans la langue source et dans la langue cible, milliseconde par milliseconde, a " $t$ " "labor"e afin de permettre une analyse de lÕinterpr"tation. En utilisant un programme sp"cialement d"velopp" < cet effet, lÕanalyse du discours de lÕinterpr"tation peut maintenant 'tre automatis"e et rendue quantitative. Les discours en langue source et en langue cible peuvent 'tre trac"s graphiquement et parall'lement sur lõaxe des temps, facilitant ainsi la comparaison entre le discours et son interpr"tation simultan"e; on peut comparer la dur"e de chaque "clat et de chaque pause, le d"but et la compensation, le degr" de simultan"it" entre lÕorateur et lÕinterpr'te et bien dÕautres param'tres importants au th"oricien de lÕinterpr"tariat.
Ce document est protégé par la loi sur le droit d'auteur. L'utilisation des services d'Érudit (y compris la reproduction) est assujettie à sa politique d'utilisation que vous pouvez consulter en ligne.

https://apropos.erudit.org/fr/usagers/politique-dutilisation/ 


\title{
Computational Discourse Analysis for Interpretation
}

\author{
sane m. yagi \\ SQU, Oman
}

\begin{abstract}
RÉSUMÉ
Une méthode informatisée qui permet l'examen attentif du discours dans la langue source et dans la langue cible, milliseconde par milliseconde, a été élaborée afin de permettre une analyse de l'interprétation. En utilisant un programme spécialement développé à cet effet, l'analyse du discours de l'interprétation peut maintenant être automatisée et rendue quantitative. Les discours en langue source et en langue cible peuvent être tracés graphiquement et parallèlement sur l'axe des temps, facilitant ainsi la comparaison entre le discours et son interprétation simultanée; on peut comparer la durée de chaque éclat et de chaque pause, le début et la compensation, le degré de simultanéité entre l'orateur et l'interprète et bien d'autres paramètres importants au théoricien de l'interprétariat.
\end{abstract}

\begin{abstract}
In order to perform discourse analysis on interpretation, a computational method that facilitates the scrutiny of SL and TL discourses on a millisecond by millisecond basis was developed. Using an in-house purpose-built program, interpretation discourse analysis can now be automated and made quantitative. Both SL and TL discourses are graphically plotted on a clock-time axis, thereby facilitating a comparison of simultaneously-delivered speech in terms of the duration of each burst and pause. As well, onsets and offsets can be compared to their equivalents in the other language, as can the degree of simultaneity of speech between speaker and interpreter, and various other matters of concern to the interpretation theoretician.
\end{abstract}

Goldman-Eisler (1968) concluded from 20 years of research that speech is an articulate and finely graded external projection of cognitive processes organized and integrated in time. Speech is the end product which reflects the workings of the mind. When speech is spontaneous, as is the case in simultaneous interpretation ( $\mathrm{SI}$ ), the relationship between the spoken word and thinking becomes more evident. Since SI consists of concurrent and semi-concurrent cognitive activities (listening, decoding, encoding, and speaking), the time factor is of crucial importance. Therefore, analyzing interpreters' time-management patterns can reveal a wealth of information about the characteristics of their performance, as well as about the cognitive processes associated with their speech.

This paper will describe a new method of discourse analysis which uses the computer to study source (SL) and target (TL) language speech on the basis of the time structures of their acoustic signals. The software that was developed for this purpose will also be reviewed. Research conclusions derived by this method will be cited to demonstrate its utility for the translation specialist and the discourse analysis linguist. 
Before describing this digital method, however, it is necessary to explain where it should be placed among the various methods of analysis used in SI research.

\section{SI METHODS OF ANALYSIS}

Research into simultaneous interpretation has used three methodologies: translation discourse analysis, cognitive experimental procedures, and psychophysiological techniques. Each of the three methodologies has a different focus, the first concerned with output speech, the second with cognitive processes producing speech, and the third with speech segments requiring intense cognitive activity.

\section{Translation Discourse Analysis}

Generalizations can be made about SI by analyzing interpreters' output and comparing it with SL discourse. Interpretation teachers and theorists alike can use discourse analysis to assess an interpreter's performance and to gain insight into the cognitive processes involved in interpretation.

There are two types of translation discourse analysis: quantitative and qualitative. In the first type, researchers reduce the data under consideration to numbers that are manipulated in several ways, using standard statistical methods. In qualitative discourse analysis, generalizations about an individual's performance and about the essence of SI are made on the basis of introspection, native speaker intuition, and subjective assessment of the degree of convergence or divergence between $S L$ and $T L$ pieces of discourse.

Different kinds of information can be obtained from these two types of discourse analysis. The quantitative method can yield information such as duration of delay, degree of simultaneity, speech burst and pause length, articulation and speech rates, etc. It can also offer the opportunity to study the influence of SL discourse temporal characteristics on TL translation. The qualitative method, on the other hand, is used to infer the possible causes of errors, and to study meaning loss, types of addition, omission, and substitution in a translation. Barik (1969) pioneered the quantitative type of translation discourse analysis but at the same time applied the qualitative method to his data.

\section{Cognitive Experimental Procedures}

Because simultaneous interpretation involves complex mental processes, several experimental tasks have been devised to study some of these individual cognitive processes (e.g. recall, recognition, split-span, shadowing, judgment, etc.). Recall tasks study the interpreter's ability to access and retrieve information from long-term memory, while recognition tasks study the ability to identify correct information. Split-span studies investigate the ability to divide attention between two tasks, and shadowing the ability to listen and speak at the same time. Judgment tasks study information encoding specificity, while SI itself is used to investigate subjects' ability to do concurrent tasks: listening and speaking, recognizing and recalling, and decoding and encoding. Many SI researchers have used these techniques (Triesman 1965; Goldman-Eisler 1972; Gerver 1971, 1976; Lambert 1983). 
Split-Span Tasks

This task type is used to inquire into selective attention, which interpreters do a lot of. It requires subjects to listen to one of two auditory signals that are presented dichotically while ignoring the other. The experimenter checks on such things as the subjects' comprehension of the attended signal, their recognition of the nature of the non-attended signal, and the interference of one signal with the other.

The relevance of this task to $\mathrm{Sl}$ is evident in its facilitation of the study of allocating attention to more than one cognitive task. Since interpreters have to divide their attention between decoding the $S L$ discourse and encoding it into the $T L$, their skill in split-span tasks is essential to good SI performance.

\section{Recall Tasks}

Recall involves searching and accessing memory to retrieve specific information. Without it, interpreters would not be able to identify the TL equivalents of the material they listen to. Recall tasks consist of an acquisition period and a test period. During acquisition time, subjects are given a list of items to remember, then are asked during test time to reproduce these items in response to a clue given by the examiner. Subjects are tested for their ability at "free recall" (recalling the items in any order), "partial recall" (recalling only some of the items), and "ordered recall" (recalling the items in the order presented).

Recall tasks are useful for studying information organization in memory, retrieval sequence, taxonomic semantic relationships, memory capacity, memory trace decay, etc. They can enlighten researchers as to how subjects store information in memory and for how long, and how they are able to access it. Recall errors are also quite valuable; confusion errors that mix up the serial order of two list items, offer a synonym in place of a list item, or paraphrase items on the list are all informative of how subjects represent information in their memory. Intrusion errors that result from guessing are indicative of the background knowledge subjects use when they fail to recall precisely.

\section{Recognition Tasks}

Subjects are presented with a list of items during an acquisition period, then are given a list during a test period that includes target items presented previously, as well as foils which function as distracters. The subjects' task is to identify targets by remembering their association to the acquisition context. Their response can be in a yes-no format, in multiple-choice, or by rank-ordering all probes according to how likely they are to be targets. Subjects often have to indicate confidence in their response using a three point scale.

Recognition tasks are mainly useful for two purposes: studying memory interference and measuring reaction times. The first makes it possible to investigate the efficiency of the interpreter's retrieval strategies, while the second is concerned with how long it takes the interpreter to access information. There is no doubt that SI lag is directly influenced by interpreter reaction times, among other things. 
Judgment Tasks

Judgment tasks are often employed to study information-encoding strategies. They are similar to recognition tasks, but generally rely on conclusions that subjects formulate rather than merely remember. After the completion of an acquisition period, the examiner might ask about which of two items was presented more recently or more frequently, which was printed in upper or lower case, which was in the native or foreign language, etc. Such questions can be informative about the degree of specificity in memory encoding.

\section{Shadowing Tasks}

Shadowing is repeating a stimulus discourse verbatim as it is being delivered. It is the task most akin to simultaneous interpretation because it shares several cognitive processes with it. Both involve listening and speaking concurrently, but the latter requires extensive decoding and language transfer.

Shadowing is used for various purposes, most important of which is reaction time. The fact that subjects have to listen to a stimulus discourse before they are able to output speech means that they maintain a reasonable delay that allows them to keep track of the source. This delay is similar to, though significantly shorter than, the delay kept by simultaneous interpreters. It is for this delay, in particular, that SI researchers use shadowing, primarily as a control task against which SI is measured.

\section{Psychophysiological Techniques}

Interpreter's perception of SL discourse segments produces arousal, an intense degree of alertness and concentrated attention. It is usually manifested in a low amplitude mental activity, some cardiovascular changes, and overt behavioural responses such as tilting the head towards the sound source. Arousal can be studied by recording the electrical activity of the brain in electroencephalograms (EEG), or by measuring cardiovascular changes using heart rate and blood pressure indices.

The size of the pupil of the eye is also thought to be an indicator of arousal and consequently of mental activity. It is fairly well-demonstrated in the literature that pupillary dilation is an index of mental effort. Some researchers, like Hess (1965), discovered that pupils dilate during the performance of mental arithmetics. Beatty and Wagoner (1978) obtained the greatest pupillary dilation in a hierarchically structured letter-matching task that required higher levels of mental processing. Matthews, et al. (1991) also found that error rate and pupil dilation amplitude rose with increased task difficulty. Just and Carpenter (1993) explored the intensity of mental processing during sentence comprehension by measuring pupillary dilation during reading. They contrasted the cognitive processing of simpler vs. more complex sentences, and found that the complex ones (object-relative center-embedded and filler-gap sentences) produced a larger change in pupil diameter. The method used for measuring pupillary dilation and employing it as an index of mental effort is called pupillometry.

Some psychophysiological methods have recently been employed to infer the mental effort associated with simultaneous interpreting. Klonowicz (1990) used cardiac activity to compare the mental workloads associated with SI and shadowing. She 
found out that both tasks elicited a cardiac mobilization, but cardiac activity soon stabilized in the course of shadowing performance, and confirmed the popular notion that SI relies on vigilant attention. She also looked for psychophysiological correlates underlying SI, and concluded that the cardiac "mobilization effect" correlates positively with reactivity temperament, trait-anxiety, and trait-curiosity.

Tommola and Niemi (1986) studied SI on-going language processes and interpreter performance quality by monitoring the size of the eye's pupil. They presented a graduate of the School of Translation Studies at the University of Turku, Finland, with five Finnish language texts, each consisting of about 400 words to translate (presumably into English). The sentence structure was manipulated in certain occasions to exhibit left-branching NP premodifications, which is relatively common in Finnish. This complication of sentence structure requires the interpreter to rearrange word order when rendering the texts into English. They discovered that pupillometry is capable of pinpointing areas of difficulty that interpreters experience in $\mathrm{Sl}$, and were able to identify the specific structures and sentences that their subject had difficulty with.

Studying SI by psychophysiological methods is a welcome development in the field. These methods introduce quantification, making $\mathrm{SI}$ a more precise discipline. Unfortunately, however, they are not without their own shortcomings.

Psychophysiological methods are not exclusively sensitive to arousal or mental effort. Pupillometrics, for example, has been used for studying mental, emotional, and sensory processes (see H ess 1972). Pupillary dilation occurs in response to more than 20 different factors. It has been used to detect morphine dependency, ${ }_{1}^{1}$ insomnia, ${ }^{2}$ fear, ${ }^{3}$ music criticism, ${ }^{4}$ etc.

These methods are of more use to SI theorists than to interpreters. Measuring the electrical activity of the brain, cardiovascular changes, and pupillary dilation can be quite intrusive. Electroencephalography requires taking X-ray pictures of the brain, while pupillometry involves the use of infrared video-pupillography. Tommola and Niemi (1986) required their subject to sit at a table and gaze at a point on a wall, with her head fixed on a head-rest to prevent head movement during translation. O bviously, such obtrusive methods have very little practical use outside the researcher's laboratory.

\section{PROPOSING A NEW METHOD OF ANALYSIS}

The above methodologies have made significant contributions to the development of SI studies. Cognitive experimental procedures dissect SI into its constituent processes and allow us to study them individually, while psychophysiological techniques probe into the nervous system to reveal the amount of mental effort required for processing specific types of information. Discourse analysis, on the other hand, offers tools for scrutinizing the actual product of Sl either to deduct the cognitive processes and strategies that produce it or to assess its quality of translation. Without these methodologies it would be difficult to formulate a cognitive theory about simultaneous interpretation, to train students to become SI practitioners, or to assess their proficiency.

Cognitive experimental procedures and psychophysiological techniques suffer, however, from two main shortcomings: they are laboratory-bound methods that focus on individual facets of SI. They are more relevant to the researchers and theo- 
reticians than to practitioners or trainees. Translation discourse analysis, on the other hand, focuses on the product as a whole and can be conducted outside the laboratory. Furthermore, it can offer feedback to interpreters that enables them to identify their areas of weakness.

The method proposed here is classified in the discourse analysis category but has the advantage of being digital, flexible and versatile. It can be used equally well in the laboratory and in the classroom, and by the translation trainer and the trainee. It can also be used as a research tool to investigate interpreter strategies, or as an assessment tool that delivers feedback on trainee performance.

\section{Digital Discourse Analysis}

Unlike traditional discourse analysis, this method relies largely on computers. Interpreters speak to the computer which in turn converts their analog speech signal to a digital signal; i.e. sound waves are transformed into a binary code that can be manipulated by computers. With the aid of an analog to digital/digital-to-analog converter, interpreters can listen to the SL discourse that the computer plays as they record their own translation. At the end of the translation session, purpose-built software displays their speech against the source signal. If automated assessment is desired, it will analyze the two signals separately, compare the translation against the source, and offer a set of performance parameters that indicate the quality of the translation. Researchers can also use this method to scrutinize the two speech signals on a millisecond by millisecond basis and thereby focus on the interpreter's time management patterns.

Digital discourse analysis makes a few assumptions that other methods do not make, but the main assumption is taken bona fide by acousticians. It assumes that the digitizing of speech signals does not lose any significant details that were in the original analog signal. The digitization of sound has now been so widely accepted that an industry has developed around producing digital sound recording systems (DAT and CD), which can both record and play back analog sound. If fidelity is a concern, most commercial software will allow users to increase their sampling rate, although for purposes of discourse analysis a low sampling rate of 100 samples per second can extract a signal's envelope and depict its time structure faithfully.

The other main assumption is that sound signals carry a lot of useful information about the linguistic content they convey. In fact, it was the widespread acceptance of this idea that made speech synthesis and speech recognition possible. What the automated assessment module of the digital discourse analysis adds is the idea that the time structure of an interpretation speech signal can reveal a lot of information about its quality. This idea developed from the commonly held view that $T L$ attempts to mirror SL discourse: the source wields influence over the development of the interpretation and the time management of the speaker affects the behaviour of the interpreter. If the speaker has a slow speech rate, for example, interpreters can cope better. Similarly, if the source material is too difficult to translate, the TL discourse may reflect that difficulty by being disjointed and/or exhibiting long pauses. Proficiency is reflected by good time management and by a high coherence with the SL discourse (see Yaghi 1994). 
Barik $(1969,1973)$ demonstrated the effectiveness of measuring speech/pause event durations as a technique for quantifying interpreters' time management behaviour. Yaghi (1994) demonstrated that the influence of SL speech events on the TL time structure (as exhibited in what he called "overlap events") can be used to assess the quality of an interpretation. Longer spans of overlap between the source speaking with the interpreter speaking (i.e. SS), and of overlaps between the source pausing with the interpreter speaking (i.e. PS), have been found to be indicative of higher degrees of simultaneity and prudence respectively and, therefore, of an interpretation's excellence. To corroborate the validity of his conclusions, Yaghi correlated these interpreter time-management parameters with marks obtained from subjective assessments and found the correlation to be quite high $(R=0.82)$.

The conclusions of discourse analysis, together with those of psychophysiological methods and cognitive experimental techniques, confirm the value of the product of interpretation in revealing the workings of the mind. Goldman-Eisler, the pioneer of psycholinguistic research, recognized the spoken discourse as an avenue to the mind when she said, "The complete speech act is a dynamic process, demanding the mobilization in proper sequence of a series of complex procedures and is the temporal integration of serial phenomena. It is a most articulate and most finely-graded external projection of internal processes organized and integrated in time" (GoldmanEisler 1968: 6). She also affirmed that,

It is only through the analysis of the act and actuality of speaking [...] that we come to grips more directly with the workings of the processes which operate when speech is being generated [...] [W] hen the utterance is spontaneous, when speakers are thinking on their feet [...] one may expect the relationship between speaking and thinking to reveal itself most naturally (Goldman-Eisler 1968: 9).

Thus, the digital discourse analysis method can be viewed not only as a precision tool for learning about discourse, but also as a research instrument for cognitive studies.

\section{Analysis Procedures}

Whatever one's conception of discourse analysis is, the digital method lends itself to it readily as it is identical to conventional discourse analysis except in the medium it uses. Traditionally, discourse analysis has been performed on spoken or written language: this method uses software that converts the sound signal to a digital signal and then to a graphic representation where peaks denote utterance and their absence, silence.

Discourse analysis of SI has tended to be concerned with the time structures of $S L$ and TL discourses. It identifies the duration of speech bursts, pauses, and delays as the focal areas of study, in addition to speech and articulation rates. Analysis compares interpreters' speech burst and pause durations and speech/articulation rates with those of speakers, and then inspects the degree of correspondence between the linguistic contents of these bursts. The latency of interpreter responses is observed, and the influence of SL speech parameters on the TL discourse is studied. Analysts then reach their conclusions about the quality of a translation and/or the mental processes that produced a certain discourse phenomenon. 
In digital discourse analysis, all of these matters can be investigated with unprecedented precision and ease. Since the spoken language can be frozen in time, exploration and manipulation are markedly simplified. The fact that the computer is used as a tool of analysis means that measurement can be exact to the millisecond. Below are the procedures used in a linguistic-based discourse analysis (procedures for assessing the quality of interpretation will be outlined in a separate paper):

(1) Once the SL and TL discourses have been recorded onto the computer, the digital discourse analysis package (ADAALab) will display discourses in the form of two speech signals; the SL in the upper half of the screen and the TL in the lower half, with the clock-time at the bottom. Each signal will consist of speech bursts represented by peaks and pauses represented by gaps.

(2) The discourse analyst can then start matching the SL bursts with their TL equivalents. The linguistic content of a speech burst is identified by playing it back, and the text then transcribed under the corresponding burst.

(3) An SL burst can be matched with its TL equivalent and be given an identical label (e.g. a number). ADAALab appends these labels to each of the bursts and encodes the match for further analysis. Pauses can be tagged in a similar way, although such coding is of little value.

(4) Analysts can also designate tag types to reflect whether a burst was accurately translated, whether there was any omission or substitution, or whether the segment was speech, pause, false start, pause filler, etc. Tag types can be entered when prompted by ADAALab.

(5) Once the tagging has been completed, ADAALab measures the duration of each speech event (pauses and bursts alike), as well as the onset and offset delays. It will also count the number of words and syllables to calculate speech and articulation rates, then tabulate the tag numbers and tag types, and extract a set of statistics that include the proportions of omission, substitution, mistranslating, false starts, and pause fillers.

To extract information about bursts, pauses, delays, speech/articulation rates, omissions, and mistranslating, ADAALab relies on a combination of user-entered factors and preprogrammed parameters. When SI discourse analysts, for example, decide to match a certain SL burst with its translation, they highlight the equivalent discourse segments and give them identical tags, thereby indicating to ADAALab that the highlighted segments are matched. Similarly, the tag type will indicate whether the segment is a burst or pause, whether it is accurately translated or mistranslated, and whether it is a false start or pause filler. On the other hand, ADAALab is preprogrammed to take the clock time at the onset of a burst or pause and deduct from the clock time at offset to determine the duration. It also automatically deducts the clock time at the onset or offset of an SL burst from the corresponding clock time at its TL equivalent. This is done in order to calculate the length of interpreter delay for that particular burst. ADAALab counts the number of words and syllables in a speech burst to compute articulation and speech rates, and is pre-programmed to generate all the statistical data of interest to SI discourse analysts (e.g. the sums, means, standard deviations, co-efficient of variation, and proportions for each discourse phenomenon).

Discourse analysts in the past used stop watches to measure small samples of burst and pause durations and interpreter delay. Now, using the digital method, each 
utterance is taken into account, and there is no room for sampling error. Furthermore, measurement is not subject to the analyst's reaction times but is consistently precise throughout.

Being automatic, ADAALab affords the luxury of finding the mean difference between an interpreter's onset and offset delays, thereby enabling the analyst to determine the degree of time control exercised in the translation. If interpreter onset and offset delays are similar in span, one can assume the interpreters are in control and managing their time efficiently, i.e. following the speaker without lagging too far behind. Longer offset delay is indicative either of difficulty in translating or verbosity in the TL.

Analysts can also use this method to determine the possible causes of errors. Since ADAALab facilitates visualizing and playing back bursts and pauses in the SL and TL discourses, it becomes easy to discover what an interpreter was doing exactly when a certain SL burst was delivered; what syllables, words, or phrases were being uttered, what SL segment the interpreter was occupied with, etc. Analysts can then infer whether enough attention resources were dedicated to listening, or if such resources were exhausted by decoding or encoding a previous SL burst. Areas of difficulty can be identified when the interpreter delay is longer than normal and the cause can be inferred from the translation context. Usually, areas of difficulty are associated with TL durations longer than their equivalents, pause fillers, false starts, droning, vowel elongation, and extended delays. What is especially interesting in such areas of difficulty is that the analyst can tell what caused them: failing to detect a keyword in the SL expression because it was delivered during an SS overlap; lagging too far behind; failing to retrieve information from the mental lexicon; or fast SL speech delivery, etc.

\section{Uses of Digital Discourse Analysis}

The aim of SI discourse analysis is to further our understanding of the phenomena of simultaneous interpretation and to assess the performance of practitioners. Digital discourse analysis, through various components of ADAALab, can be used effectively for both purposes.

With digital discourse analysis, several theoretical questions can be investigated. Complex grammatical structures, for example, appear to slow down some interpreters. Whether this is a universal phenomenon is not clearly documented in the literature. Technical terminology, metaphorical expressions, and numerical figures are thought to be irritants to interpreters, but to what extent do these detract from their performance? When can they break down the translation process? Do all interpreters have the same collapse point? Does interpreter performance differ from one language combination to another? In what quantitative ways does proficiency manifest itself in a translation? All these questions and more can be probed with the aid of digital discourse analysis.

Theorizing about SI can be made more empirical with this type of analysis. By controlling some SL parameters and varying others, investigators can see their effect on interpreter performance. High SL articulation and speech rates, for example, have often been cited by practitioners as sources of difficulty. Investigators can now study the extent of these hindrances by manipulating the tempo of a stimulus SL discourse 
to either slow down or speed up. They can then offer both the tempo-altered and non-altered discourses to two groups of subjects for translation. By comparing the subjects' performances, the effect of speech delivery tempo becomes evident.

Yaghi (1994) investigated the effect of SL speech delivery rates using discourse samples with conventional themes and language that were delivered at an exceptionally low speech rate ( 3.7 syllables/second as opposed to the natural rate of $4.8 \mathrm{~s} / \mathrm{s}$ ). Digital discourse analysis found that there was a drastic reduction in omission proportions, a deceleration in TL speech delivery rates, and a reduction in the duration of onset and offset delays. Moreover, the spans and proportions of overlap events changed considerably, resulting in longer duration SP, PS, and PP, although SS re mained constant in mean size. Their overall proportions became roughly similar, with PP commanding the largest fraction and SS the smallest. Unlike natural delivery rates, low SL speech rates resulted in the simultaneity measure (SS) occupying onefifth of the discourse time compared to more than two-fifths in natural discourses. In the time dissipation measure (PP), the SS occupied more than one quarter of the discourse time compared to one tenth in natural speech discourse. Despite these changes, SS and PS continued to be reliable measures of performance when the speech delivery rate was exceptionally low.

In terms of performance assessment, digital discourse analysis has been applied and proven to be indispensable. Yaghi (1994) used it to identify measures of translation quality, applying it to a 180-minute translation discourse rendered by six subjects of varying degrees of proficiency who were shadowed by six other bilinguals. Two quantitative methods were used for assessment: one studied the time structures of SL and TL signals, while the other analyzed similarities and differences between and within the two signals using signal correlation techniques. The findings are indicative of the wealth of information that can be obtained with digital discourse analysis.

Yaghi (1994) also studied the status of both SL and TL signals along a clock-time axis that described any changes in speech events as four states he called "overlap events": when the speaker is talking and the interpreter is pausing (SP), when both are speaking (SS), when the speaker is pausing while the interpreter is speaking (PS), and when both are pausing (PP). These four overlap events account for all possible states of speaking and pausing that concurrently occur in the SL and $T L$ discourse. Since they reflect how interpreters manage their time in relation to the SL time structure, these overlap events have also been referred to as time management patterns.

Time management patterns expressed in overlap events are quite distinct from one another and are indicative of interpreter proficiency. Proficiency has been found to be characterized by: (i) a minimization of time dissipation through adherence to very short PP spans (around one third of a second in duration) and very small overall PP proportions (about 7\% of the discourse time); (ii) a minimization of interpreter inactivity by adherence to short SP spans (around three quarters of a second) and small overall SP proportions (about one quarter of the discourse time); (iii) a maximization of prudence, i.e. long PS (more than half a second in duration and about one fifth of the proportional discourse time); and (iv) a maximization of simultaneity by keeping long SS spans (more than one second in duration and about half of the discourse time in proportion). 
The relationship between linguistic-based measures of performance and time management patterns is not a direct relationship because they reflect different facets of interpreter behaviour. N evertheless, digital discourse analysis has shown that individual time management parameters can be indicative of at least one linguistic-based measure each: they are capable of indicating the degree of omission, length of delay and speech rate in a translation discourse. It has been determined, for instance, that a pause shared by both speaker and interpreter is a time-dissipation measure which has an inverse relationship with delay. Because PP is indicative of expendable time, and a delay connotes time shortage, there is an inverse relationship between them. The longer both discourse participants are passive, the shorter interpreter delays are; i.e. if interpreters lag too far behind, they cannot afford long spans of pausing concurrent with speaker pauses. This conclusion validates the notion that SL pauses are especially valuable in the SI process. Furthermore, it corroborates Barik's (1969) assertion that SL pauses are used to reduce interpreter delays.

On the other hand, PS (when interpreters speak during speakers' pauses) is a measure of prudence. Digital discourse analysis has shown that PS correlates negatively with omission and delay; the longer interpreters talk during a speaker's pause, the shorter their delays are and the less they exhibit omission in their translation. Being confident and translating at the same time as the speaker is talking (SS) is viewed as a measure of simultaneity; SS has been found to correlate positively with omission. The longer the span of speaking simultaneously with the SL speaker, the greater the chance an interpreter will make omissions.

The time management patterns extracted by digital discourse analysis have also been compared with subjective assessments. Yaghi (1994) solicited qualitative assessments of the 180 minute translation corpus from 47 experts, and carried out a multiple regression analysis of the mean time-management pattern durations against the subjective assessment marks. The analysis showed a very strong correlation coefficient $(R=0.82)$. In fact, by using the regression equation, it is possible to predict with some confidence $\left(R^{2}=.672\right)$ the mean mark a translation may receive by subjective assessment.

\section{CONCLUSION}

The results reported here are only a small illustration of the power digital discourse analysis gives to the interpretation specialist. With the right experimental procedure, a wealth of empirical conclusions can be drawn. It is a method providing concrete results that may offer much guidance to theories of simultaneous interpretation.

\section{NOTES}

1. Navarro et al. 1992.

2. Lichstein et al.1992.

3. Sturgeon et al. 1989.

4. Mudd et al. 1990. 


\section{REFERENCES}

Barik, Henri C. (1969): A Study of Simultaneous Interpretation, Unpublished doctoral dissertation, University of North Carolina at Chapel Hill.

- - (1973): "Simultaneous Interpretation: Temporal and Quantitative Data", Language and Speech, 16, pp. 237-270.

Beat $t y$, Jackson and Brennis L. Wagoner (1978): "Pupillometric Signs of Brain Activation Vary with Level of Cognitive Processing", Science, 199, pp. 1216-1218.

Gerver, David (1971): Simultaneous Interpretation and Human Information Processing, Unpublished doctoral dissertation, Oxford University, Oxford.

- - (1976): "Empirical Studies of Simultaneous Interpretation: A Review and a Model", in Richard W. Brislin (Ed.), Translation Applications and Research, N ew York, Gardner Press.

Gol dman-Eisl er , F. (1968): Psycholinguistics: Experiments in Spontaneous Speech, London, Academic Press.

- - (1972): "Segmentation of Input in Simultaneous Interpretation", J ournal of Psycholinguistic Research, 1, pp. 127-140.

H ess, E. H. (1965): "Attitude and Pupil Size", Scientific American, 212, pp. 46-54.

- - (1972): "Pupillometrics: a M ethod of Studying Mental, Emotional, and Sensory Processes", in N. S. G r een fiel d and R. A. St r en bach (Eds.), Handbook of psychophysiology, New York, Holt, Rinehart, and Winston, pp. 491-534.

Just, Marcel A. and Patricia A. Carpenter (1993): "The Intensity Dimension of Thought: Pupillometric Indices of Sentence Processing", Canadian Journal of Experimental Psychology, 47-2, pp. 310-339.

KI on ow icz, Tatiana (1990): "The Interaction of Individual Differences and Mental Workload", Polish Psychological Bulletin, 21-1, pp. 37-48.

Lambert, Sylvie Michele (1983): Recall and Recognition among Conference Interpreters, Unpublished doctoral dissertation, University of Sterling.

Lichstein, Kenneth L., Ronald S. Johnson, Sunil S. Gupta, Deborah L. O'Laughl in et al. (1992): "Are Insomniacs Sleepy During the Day? A Pupillometric Assessment", Behavior Research and Therapy, 30-3, pp. 283-292.

M at thews, G., W. M iddl et on, B. Gil mart in and M. A. Bullimore (1991): "Pupillary Diameter and Cognitive Load", Journal of Psychophysiology, 5-3, pp. 265-271.

M udd, Samuel, Christine G. Con way and Diane E. Sch indl er (1990): "The Eye as M usic Critic: Pupil Response and Verbal Preferences", Studia Psychologica, 32 (1-2), pp. 23-30.

N avar r O, M iguel and Jose M. Pr iet o (1992): "A New Psycho-Biological Test to Detect MorphineDependent Users in the Work Environment", Applied Psychology: An International Review, 41-2, pp. 175-184.

St ur geon, Robert S., Leslie M. Cooper, and Robert J. Howell (1989): "Pupil Response: A Psychophysiological Measure of Fear During Analog Desensitization", Perceptual and M otor Skills, 69 (3, Pt. 2), pp. 1351-1367.

Tommol a, Jorma and Pekka N iemi (1986): “M ental Load in Simultaneous Interpreting: An OnLine Pilot Study", in Lars Sigfred Evensen (Ed.), Nordic Research in Text Linguistics and Discourse Analysis, Norway, Tapir Publishers.

Tr eisman , A. M . (1965): “The Effects of Redundancy and Familiarity on Translating and Repeating Back a Foreign and a Native Language", British Journal of Psychology, 56, pp. 369-379.

Yaghi, Hussein M. (1994): A Psycholinguistic Model for Simultaneous interpretation, and Proficiency Assessment by Automated Acoustic Analysis of Discourse, Unpublished doctoral dissertation, Auckland University.

Yagi, Sane M . and M. J. Yagh i (1996): ADAALab ${ }^{\mathrm{TM}}$, v.1.0.6. Linguistic Software Series, Auckland, New Zealand, DamienTech.

Yagi, Sane M. and Ali Benmer zou ga (Forthcoming): "Statistical Models for Assessing Simultaneous Interpretation", Assessment and Evaluation in Higher Education. 